\title{
Reflexões Sobre O Brincar Como Promotor Do Desenvolvimento Integral Da Criança Com Transtorno Do Espectro Autista
}

\author{
Francisca Francisete de Sousa Nunes Queiroz ${ }^{1}$, Christina César \\ Praça Brasil' ${ }^{1}$, Fabiana Neiva Veloso Brasileiro ${ }^{2}$, Felícia Gabler ${ }^{2}$, Erick \\ Tomé de Lima ${ }^{3}$ e José Eurico de Vasconcelos Filho ${ }^{3}$
}

1 Programa de Pós-Graduaçao em Saúde Coletiva, Universidade de Fortaleza, Brasil | zete.queiroz@hotmail.com; cpraca@unifor.br | https://orcid.org/0000-0002-2933-4974; https://orcid.org/0000-0002-7741-5349

${ }^{2}$ Curso de Graduação em Psicologia - Universidade de Fortaleza, Brasil fabianaveloso@unifor.br; feliciagabler@icloud.com | https://orcid.org/0000-0002-31704268; https://orcid.org/0000-0001-7381-0600

\begin{abstract}
${ }^{3}$ Núcleo de Aplicação em Tecnologia da Informação - NATI - Universidade de Fortaleza, Brasil | ericktlima@edu.unifor.br; euricovasconcelos@unifor.br | https://orcid.org/0000-0002-9922-1684; https://orcid.org/0000-0002-68810814
\end{abstract}

\begin{abstract}
Resumo: Introdução: O ato de brincar perpassa os diferentes níveis de desenvolvimento da criança, sendo fundamental para o bem-estar social, emocional, cognitivo e físico. Crianças com Transtorno do Espectro Autista - TEA podem apresentar dificuldades em diversas áreas do desenvolvimento. É importante que profissionais da saúde, familiares, cuidadores e educadores reflitam sobre o papel do brincar na mediação das relações e das trocas, que favorecem o desenvolvimento dessas crianças. Objetivo: Identificar estudos que propiciem reflexões sobre a relevância do brincar para a promoção do desenvolvimento integral de crianças com TEA. Método: Realizou-se uma revisão integrativa, de fevereiro de 2019 a outubro de 2020, nas principais bases de dados da saúde. O material selecionado foi analisado qualitativamente, com base nos objetivos e resultados dos estudos, fazendo-se emergir duas temáticas. Resultados: As temáticas "reflexões teóricas sobre o brincar" e o "ato de brincar no contexto do desenvolvimento da criança com TEA" trouxeram reflexões sobre a importância do brincar, a partir de diferentes perspectivas teóricas, atestando que o ato de brincar é essencial para o desenvolvimento da criança com TEA. Isto contempla várias possibilidades de interação com pessoas e realidades. Conclusões: A partir das evidências apresentadas na literatura, o ato de brincar deve ser incentivado no contexto de vida pessoal e terapêutico de crianças com TEA, oferecendo aos profissionais da saúde e aos familiares alternativas de estimulação global dessa população, além de favorecer o desenvolvimento das habilidades de comunicação, interação social e motora.
\end{abstract}

Palavras-chave: Desenvolvimento Infantil; Transtorno Autístico; Criança; Jogos e Brinquedos.

\section{Reflections on Playing as a Promoter of the Integral Development of Children with} Autistic Spectrum Disorder

Abstract: Introduction: The act of playing permeates the different levels of the child's development, and is fundamental for social, emotional, cognitive and physical well-being. Children with Autistic Spectrum Disorder - ASD may experience difficulties in several areas of the development. It is important that health professionals, family members, caregivers and educators reflect on the role of playing in mediating relationships and exchanges, which favours the development of these children. Objective: Identify studies that provide reflections on the relevance of playing for the promotion of integral development of children with ASD. Method: An integrative review was carried out, from February 2019 to October 2020, in the main health databases. The selected material was qualitatively analysed, based on the objectives and results, making two themes emerge. Results: The themes "theoretical reflections on playing" and "the act of playing in the context of the ASD children's development" brought reflections on the importance of playing, from different theoretical perspectives, attesting that the act of playing is essential for the development of children with ASD. This contemplates a large number of possibilities for interaction with people and realities. Conclusions: Based on the evidence presented in the literature, the act of playing should be encouraged in the context of the personal and therapeutic life of children with ASD, offering health professionals and family members alternatives for global stimulation of this population, in addition to favouring the development of children's communication, social and motor interaction skills.

Keywords: Child Development; Autistic Disorder; Child; Play and Playthings. 


\section{Introdução}

O Transtorno do Espectro Autista (TEA) é uma condição do neurodesenvolvimento, que se apresenta nos primeiros anos de vida da criança (American Psychiatric Association [APA], 2014). Aspectos etiológicos ainda são desconhecidos, embora pesquisas mostrem correlatos neurobiológicos e genéticos (Loke, Hannan, \& Craig, 2015; Maia et al., 2018).

Pesquisas mostram o aumento da prevalência de casos TEA na população mundial, incluindo o Brasil (Fombonne, 2018; Fuentes, Hervás, \& Howlin, 2020). Isto pode estar relacionado a maior conscientização sobre essa condição, aos critérios e ferramentas de diagnóstico e a melhor comunicação (Araújo, Veras, \& Varella, 2019). A World Health Organization (WHO) aponta que uma a cada 160 crianças tem TEA, estimando que $1-2 \%$ das crianças em todo o mundo tenham o diagnóstico, o que corresponde a aproximadamente 52 milhões de pessoas (WHO, 2019).

O desenvolvimento infantil é definido como um processo multidimensional e integral, que se inicia a partir da concepção, englobando o crescimento físico, a maturação neurológica, o desenvolvimento comportamental, sensorial, cognitivo e de linguagem (Brasil, 2016). Em contrapartida, alterações no desenvolvimento assumem relevância nas sociedades atuais, e crianças com esse tipo de acometimento são consideradas mais vulneráveis e requerem suporte específico.

Autores destacam que o brincar se relaciona com os diversos níveis de desenvolvimento da criança (Figueiredo, Gatinho, Torres, Pinto, Santos, \& Veríssimo, 2015), sendo o principal potencializador do desenvolvimento infantil. Nessa compreensão, o brincar é fundamental para o bem-estar social, emocional, cognitivo e físico (Vieira, 2018).

Crianças com TEA podem apresentar dificuldades no desenvolvimento da interação social, da comunicação e motor. Muitas também apresentam comportamentos que prejudicam a experiência do brincar, visto que se limitam, na maioria das vezes, a repetições ritualísticas, brincar isolado e disfuncional, não permitindo a interferência e a participação de outras pessoas (Calegari, Luz, Campos, Borba, \& Ribeiro, 2018).

Leal, Gradim, \& Souza, (2020) relatam uma intervenção com base em atividades lúdicas para o treino de habilidades sociais em crianças com TEA, a qual foi realizada por cinco meses, com oito crianças de 3 a 5 anos. Observaram que o uso desse recurso foi um fator essencial no treino de habilidades sociais em todas as crianças.

Quanto ao envolvimento da família no ato de brincar, uma pesquisa realizada no Reino Unido, com 306 pais, mostrou que os respondentes informaram que eram principalmente ou igualmente responsável pelas rotinas diárias dos cuidados com seus filhos, e que passavam mais tempo brincando do que realizando qualquer outra atividade, embora quase a metade não tivesse recebido treinamento para tal (Potter, 2017).

Diante de dois cenários, o primeiro, em que os profissionais mediam o brincar; e, o outro, em que os familiares se envolvem e conduzem as brincadeiras, percebe-se que esse tipo de atividade é usado tanto no processo terapêutico, como também faz parte da rotina diária das famílias de crianças com TEA, auxiliando na estimulação dessas crianças.

Dentre as lacunas para a compressão do brincar como uma abordagem necessária a promoção do desenvolvimento de crianças com TEA, o presente estudo justifica-se pela necessidade de obtenção de uma base teórica para o melhor entendimento sobre o assunto. Assim, objetivou-se identificar estudos que propiciem reflexões sobre a relevância do brincar para a promoção do desenvolvimento integral de crianças com TEA. 


\section{Metodologia}

Existem vários caminhos para refletir sobre a produção do conhecimento. Neste estudo, realizou-se uma revisão integrativa $(\mathrm{RI})$ para verificar a produção científica a partir de pesquisas qualitativas. A RI permite a investigação criteriosa e sistematizada sobre determinado problema, objetivando identificar eventuais lacunas do conhecimento científico (Mendes, Silveira, \& Galvão, 2008). Nessa perspectiva, busca-se o aprofundamento sobre o assunto, no intuito de ampliar os referenciais teóricos e respaldar a pesquisa, favorecendo o domínio sobre a temática e trazendo elementos relevantes para a construção do conhecimento.

Percorreram-se cinco etapas para a realização desta RI: estabelecimento da questão norteadora; seleção e obtenção dos artigos (critérios de inclusão e exclusão); avaliação dos estudos pré-selecionados; discussão dos resultados; e apresentação dos resultados da revisão (Sousa, Silva, \& Carvalho, 2010).

$\mathrm{Na}$ primeira etapa, estruturou-se a seguinte questão norteadora: o que a literatura evidencia sobre o ato de brincar para o desenvolvimento integral da criança com TEA?

De fevereiro de 2019 a outubro de 2020, selecionaram-se os textos, por meio da busca na literatura científica, nas principais bases de dados e portais da área da saúde (Biblioteca Virtual em Saúde - BVS, CAPES, EBSCOhost, Google Scholar, Scientific Electronic Library Online - SciELO, Science Direct, Researchgate, Redalyc, e BDTDibict e livros). Para isso, utilizaram-se os seguintes descritores e seus correspondentes, em português e inglês indexados nos Descritores em Ciências da Saúde (DeCS): habilidades (skills), desenvolvimento infantil (child development), brincar/brincadeiras e brinquedos (play and playthings), atividades lúdicas (play activities) e transtorno do espectro autista (autistic disorder). Foram realizadas todas as possibilidades de cruzamento entre os descritores selecionados, por meio de duas estratégias de busca (A e B). As palavras foram cruzadas utilizando-se os operadores booleanos "AND", na estratégia A, e "OR", na estratégia B.

Os critérios de inclusão foram: textos publicados de 2010 a 2019, que abordam o brincar para o desenvolvimento infantil, incluindo conceitos, metodologias, intervenções que usem o brincar para estimular crianças com TEA; e textos que contemplem a questão norteadora. Foram excluídos os textos que não apresentavam conteúdo relevante para a temática; que incluíam outros grupos, como idosos e adultos; e os que não referenciavam as crianças com TEA.

A análise crítica dos textos pré-selecionados foi realizada a partir da leitura aprofundada, bem como da interpretação. A análise foi predominantemente de cunho qualitativo, considerando-se os objetivos e os resultados dos estudos, interpretando-se os sentidos das ideias centrais das teorias que abordam o ato de brincar no contexto da criança com TEA. Para isso, utilizou-se a análise de conteúdo na modalidade temática (Minayo, Deslandes, \& Gomes, 2013). Esse método possibilita um maior conhecimento sobre o assunto em pauta dentro de um contexto específico (Minayo, 2014).

Em síntese, realizou-se a identificação das ideias centrais dos textos, passando pela interpretação dos sentidos e agrupamento dos pontos relevantes em duas temáticas de análise.

\section{Resultados e Discussão}

Como resultado, identificaram-se 108 produções científicas que contemplam o tema, sendo 100 artigos, 5 dissertações e teses, e 3 livros (Tabela 1). 
Tabela 1. Pesquisa de revisão bibliográfica com relação ao quantitativo de publicações associado a base de dados e ao tipo.

\begin{tabular}{lcc}
\hline \multicolumn{1}{c}{ Dados/Portais/Repositórios } & N & Tipo \\
\hline BVS & 2 & Artigos \\
CAPES & 23 & Artigos \\
EbscoHost & 27 & Artigos \\
Researchgate & 2 & Artigos \\
Redalyc & 7 & Artigos \\
Google Scholar & 31 & Artigos \\
Science Direct & 5 & Artigos \\
Scielo & 3 & Artigos \\
BDTDibict & 5 & Dissertações e teses \\
Livros & 3 & Livros \\
Total & $\mathbf{1 0 8}$ & Estudos \\
\hline
\end{tabular}

Dentre as publicações listadas na tabela 1, excluíram-se aquelas em que, mesmo presente o descritor pesquisado, após a leitura, verificou-se que não abordavam o brincar para o desenvolvimento da criança com TEA (74). Mantiveram-se para análise 34 artigos. Assim, criou-se uma tabela para melhor explorar os objetivos e resultados dos estudos inclusos. Dessa forma, definiram-se duas temáticas de análise, quais sejam: reflexões teóricas sobre o brincar; e ato de brincar no contexto do desenvolvimento da criança com TEA.

\subsection{Reflexões Teóricas Sobre o Brincar}

São diversas perspectivas teóricas que discutem o brincar, variando conforme as perspectivas filosóficas e características do contexto social, histórico e cultural que sustentam suas ideias, concepções e pontos de vista. Segundo Souza, (2019), a relação da criança com o mundo é mediada e o brincar possui diferentes significações e construções que sofrem variações com o tempo.

Apesar de existir uma diversidade de teorias, consideram-se relevantes para compreensão científica aquelas que facilitam o entendimento sobre desenvolvimento infantil. Portanto, mencionam-se alguns renomados autores, que mesmo com visões um pouco distintas, consideram que o brincar é fundamental para o desenvolvimento da criança, como: Piaget, (1978), Ferland, (2006), Vygotsky, (2008) e Huizinga, (2019).

$\mathrm{Na}$ antiguidade, a atividade lúdica não estava ligada unicamente à infância, mas às pessoas em geral (Moraes, 2012). O filósofo Platão falava da formação moral do cidadão a partir da infância, por meio de brincadeiras. Desde então, outros estudiosos, antropólogos, filósofos, psicólogos e educadores empenharam esforços teóricos e práticos para valorizar o papel do brincar no desenvolvimento humano, sobretudo na infância (Ferreira, 2010).

Para Piaget, (1978), o brincar assume um papel fundamental nas etapas de desenvolvimento da criança. É nessa fase do desenvolvimento que são caracterizadas diferentes formas do indivíduo interagir com a realidade e organizar seus conhecimentos visando sua adaptação. Dessa forma, desde criança, o indivíduo vai construindo seu desenvolvimento mental, levando em consideração as áreas motora, intelectual e afetiva.

Santos, (2016), enfatiza que o brincar traz possibilidades de construir conhecimento e levar ao desenvolvimento cognitivo, sendo o principal meio utilizado pela criança para desenvolver suas potencialidades e habilidades, de acordo com o contexto e a idade. 
Para Vygotsky, (2008), o brincar é visto como uma maneira de interpretar e assimilar o mundo. Teoriza que, ao brincar, a criança constrói situações, desenvolve o pensamento abstrato e o amadurecimento de regras sociais. A partir da imitação e da representação, a criança promove e facilita a interação social, iniciando a apropriação das normas e comportamentos sociais.

Em conformidade, os estudos analisados contribuem com as reflexões sobre o brincar, salientando que esse ato leva as crianças a atribuírem significado às situações vivenciadas e adquirem, de forma ativa, novos conhecimentos sobre objetos, pessoas e acontecimentos. Tudo isso ocorre por meio da integração de novas experiências, considerando o que já é familiar (Santos, 2016).

Hiuzinga, (2019) aponta que o brincar é uma atividade repleta de sentido, uma ocupação vital. Segundo o autor, brincar implica em interessar-se pelo desconhecido, envolvendo uma série de atividades físicas, mentais sociais, comunicativas e emocionais, fundamentais ao desenvolvimento humano.

Aguiar, (2018) aponta que o lúdico (ludus) abrange jogos infantis, recreação, divertimento, brincadeira, brincar, competições, representações litúrgicas e teatrais, e jogos de azar. O lúdico é a essência do ser humano, pois, antes de se tornar Homo Sapiens, quer dizer, um ser pensante, e, Homo Faber, aquele que faz; ele se constitui como Homo Ludens, um ser lúdico, que brinca, joga e interage de forma lúdica conforme a sua realidade.

Ferland, (2006) reafirma que, ao brincar, a criança progride nas diferentes esferas do seu desenvolvimento. A partir da brincadeira, a criança vincula o que é familiar ao desconhecido, e, a partir dessa conexão, ela consegue construir a sua realidade. $\mathrm{O}$ autor ressalta que a definição do brincar ainda é uma incógnita, e, mesmo tendo vários conceitos, é unanime sua repercussão no desenvolvimento integral do sujeito.

Alguns fatores podem dificultar o engajamento da criança ao ato de brincar, tais como: ter pouca ou nenhuma convivência com outras crianças, estar inserida em um ambiente restrito de estímulos ou não privilegiar o tempo de brincar. Isto mostra a importância de oferecer condições que favoreçam o brincar para todas as crianças, mesmo aquelas com algum transtorno ou deficiência (Albuquerque \& Benitez, 2020).

$O$ ato de brincar, além de evidenciar possíveis déficits, possibilita identificar e acompanhar algumas alterações no desenvolvimento, o que inclui o TEA. Para as pessoas com autismo, o brincar não é aprendido de forma espontânea e/ou por observação, é necessário introduzir o brincar, a partir de estímulos, respeitando suas demandas e especificidades (Cipriano \& Almeida, 2016).

\subsection{O Ato de Brincar no Contexto do Desenvolvimento da Criança com TEA}

O autismo configura-se como um transtorno do neurodesenvolvimento, com origem neurobiológica que compromete regiões específicas para o funcionamento cerebral, levando o sujeito a dificuldades diversas para o desenvolvimento satisfatório de suas habilidades. Assim é descrito pelo Diagnostic and Statistical Manual of Mental Disorders, da American Psychiatric Association; o DSM - V, que está em sua quinta edição; e apoiado pelo Código Internacional de Doenças, décima edição - CID-10 (APA, 2014).

O TEA apresenta prejuízos na comunicação e na interação social (déficits de reciprocidade social e emocional, nos comportamentos e compreensão do uso da comunicação nãoverbal e na formação e manutenção de relações adequadas ao nível de desenvolvimento); além de comportamentos restritos e repetitivos (estereotipias motoras, ecolalia, manipulação de objetos, fixação à rotina, interesses restritos e excessivos, hiper ou hiporreação a estímulos sensoriais), limitando ou desabilitando o indivíduo para o funcionamento diário (Griesi-Oliveira \& Sertie, 2017). 
Nesse sentido, o brincar, em suas propostas lúdicas, vem contemplar o grande número de manifestações do TEA, buscando atender e estimular cada criança em suas particularidades; mesmo que para algumas delas o ato de brincar esteja comprometido. Observa-se, portanto, que o nível de desenvolvimento físico, cognitivo e psicológico das crianças vai influenciar a prática do lúdico (Cipriano \& Almeida, 2016).

No intuito de estimular as crianças com TEA e buscar a melhoria das habilidades comunicacionais, sociais e motoras, o 'brincar' constitui-se como atividade essencial, visto que é inerente ao ser humano e fundamental para o desenvolvimento infantil. No âmbito do TEA, o ato de brincar é também considerado um recurso terapêutico, relacional e lúdico (Calhoca, 2019).

Fala-se frequentemente do brincar relacionado ao desenvolvimento da criança. Contudo, diversas abordagens teóricas, descrições e definições, voltados tanto à educação como à saúde, chegam a confundir os termos 'brincadeira, brinquedo e jogos'. Estes, porém, são definidos com base nas características das atividades, situações e comportamentos dos indivíduos e grupos em um dado período e contexto social (Fonseca \& Da Silva, 2015). Portanto, destaca-se que o termo 'brincar' se refere a um somatório de saberes, que não comportam uma única definição.

Sob um aspecto mais abrangente e com base em interpretações de estudos brasileiros, a 'brincadeira' é vista como a ação de brincar. $O$ 'jogo' pode ser considerado uma brincadeira com a presença de regras. Já o 'brinquedo' é o objeto com o qual as crianças brincam e interagem (Clemente, 2019). A mesma ideia é defendida por Cipriano e Almeida, (2016), em que o brincar abrange a brincadeira e o jogo, sendo ambos amparados pelo brinquedo, como objeto que dá suporte à ação brincante.

Alves e Bianchin, (2010) indicam que a palavra 'jogo' significa diversão, ou uma brincadeira. É um recurso capaz de promover um ambiente organizado, motivador, agradável e enriquecido, possibilitando a aprendizagem de várias habilidades. Para Ramos, Lorenset, \& Petri, (2016), o 'jogo' é uma atividade com valor recreativo orientada por um ou mais jogadores que disputam entre si, no intuito de alcançar metas, por meio de regras e restrições previamente definidas. Essa organização e objetivação que os jogos propiciam favorecem o direcionamento das atividades de crianças com TEA, pois, nessa perspectiva, é possível imprimir normas, sequências e estabelecer objetivos a serem alcançados, o que facilita, em termos terapêuticos, acompanhar a evolução.

Os brinquedos também têm um papel fundamental, pois levam a criança a interagir ao brincar, o que deve ser estimulado nas crianças com TEA. Esses objetos, porém, precisam adequar-se ao interesse, às necessidades e às capacidades da etapa de desenvolvimento. Nesse sentido, objetos, sons e espaços podem virar brinquedo, por meio da interação com a criança. Importante destacar que os brinquedos representam o princípio de muitas brincadeiras, sendo um recurso facilitador para crianças com TEA (Saviani \& Pinheiro, 2014).

Diante de diversas conjecturas sobre o brincar, identifica-se que este ato contribui para a criança desenvolver diversas habilidades. $O$ brincar é também considerado importante para profissionais que trabalham com crianças e para o aumento do envolvimento dos pais e/ou familiares com seus filhos. Aliado a isso, os estudos de Lee, Xu, Guo, Gilic, Pu, \& Xu, (2019) e Orr, (2020) mostram os vários benefícios do brincar para as crianças com TEA, quais sejam: favorece a formação de vínculos afetivos; propicia o estabelecimento e o aprofundamento das relações; amplia a comunicação e a compreensão; melhora a expressão de sentimentos e insatisfações; e possibilita maior qualidade de vida.

Diante do exposto, observa-se que a influência do brincar é relevante para o desenvolvimento de habilidades essenciais para as crianças com TEA. Nesse sentido, novas maneiras de estimular as habilidades estão sendo estudadas em pesquisas multidisciplinares que incluem profissionais da tecnologia, da educação e da saúde, no intuito de transformar a relação entre a tecnologia e o brincar cada vez mais favorável a evolução das crianças com TEA (Aragão, Bottentuit Junior, \& Zaqueu, 2019). 


\section{Conclusões}

As temáticas discutidas trazem uma reflexão teórica sobre o brincar, e reforçam que esse ato estimula o desenvolvimento integral de todas as crianças, considerando os níveis cognitivo, pessoal, social, afetivo, motor, linguístico e sensorial. Por isso, o brincar é um recurso que traz importantes benefícios ao desenvolvimento integral das crianças com TEA, devendo ser estimulado de forma direcionada, nos ambientes familiar, educacional e terapêutico.

A caracterização dos termos 'brincadeira', 'jogo' e 'brinquedo' e as reflexões que ajudam a relacionar esses conceitos ao processo do desenvolvimento infantil auxiliam o terapeuta e os familiares no desenvolvimento dessas atividades, chamando a atenção para a função de cada um no processo de estimulação da criança com TEA.

Pode-se afirmar que o brincar, ao acontecer na forma de brincadeira e/ou jogo, tendo ou não brinquedo como o suporte material, traduz-se como recurso, estratégia, instrumento, ação, possibilidade e intervenção promotora do desenvolvimento junto a crianças com TEA.

A relevância deste estudo, no âmbito da pesquisa qualitativa, reflete a significância das revisões de literatura para elucidar questões que associam o brincar ao desenvolvimento de crianças com TEA, elucidando teorias e estratégias que favorecem ao terapeuta a ampliação do embasamento teórico sobre o assunto.

\section{Referencias}

Aguiar, J. F. (2018). O lúdico é um saber? Vozes docentes sobre lúdico na docência do ensino superior. [Dissertação de Mestrado em Educação, Universidade Federal do Rio de Janeiro UFRJ, Rio de Janeiro, Brasil].

https://ppge.educacao.ufrj.br/disserta\%C3\%A7\%C3\%B5es2017/dJonathanAguiar.pdf

Albuquerque, I. \& Benitez, P. (2020). O brincar e a criança com transtorno do espectro autista: revisão de estudos brasileiros. Revista Ibero-Americana De Estudos Em Educação, 15(4), 1939-1953.

Alves, L. \& Bianchin, M. A. (2010). O jogo como recurso de aprendizagem. Rev. psicopedag., São Paulo, 27(83), 282-287

American Psychiatric Association. (2014). Diagnostic and Statistical Manual of Mental Disorders (5th ed.). Arlington, VA: American Psychiatric Publishing.

Aragão, M. C. M., Bottentuit Junior, J. B. \& Zaqueu, L. C. C. (2019). O uso de aplicativos para auxiliar no desenvolvimento de crianças com transtorno do espectro autista. Olhares \& Trilhas, Uberlândia, 21 (1), p. 43-57.

Araújo, J. A. M. R., Veras, A. B. \& Varella, A. A. B. (2019). Breves considerações sobre a atenção à pessoa com transtorno do espectro autista na rede pública de saúde. Rev. Psicol. Saúde, 11 (1), 89-98.

Brasil. Ministério da Saúde. Secretaria de Atenção à Saúde. (2016). Diretrizes de estimulação precoce: crianças de zero a 3 anos com atraso no desenvolvimento neuropsicomotor. Ministério da Saúde, Secretaria de Atenção à Saúde. Brasília: Ministério da Saúde.

Calegari, T., Luz, J.H., Campos, Y.A., Borba, R. \& Ribeiro, C. (2018). A criança autista em sessão de brinquedo terapêutico dramático: uma análise winnicottiana. Rev Soc Bras Enf Ped.,18 (1), 43-8.

Calhoca, C. A. R. (2019). A atividade lúdica da criança: o brincar em crianças com desenvolvimento atípico. 2019 [Dissertação de Mestrado em Educação Pré-escolar, Instituto Politécnico de Lisboa-Escola Superior de Educação de Lisboa, Lisboa]. https://repositorio.ipl.pt/bitstream/10400.21/10420/1/Relat\%C3\%B3rio_PPS_II_Catarina_Calh oca.pdf

Cipriano, M. S. \& Almeida, M. T. P. (2016). O brincar como intervenção no transtorno do espectro do autismo. Extensão em Ação, Fortaleza, 2 (11), 77-91. 
Clemente, C. A. P. (2019). Jogos e brincadeiras como recursos pedagógicos na educação préescolar e 1. ciclo do ensino básico. [Dissertação de Mestrado em Educação Pré-escolar, Universidade do Minho - Instituto de Educação, Braga]. http://repositorium.sdum.uminho.pt/bitstream/1822/63435/1/Cl\%c3\%a1udia\%20Andreia\%20P inheiro\%20Clemente.pdf

Ferland, F. (2006). O modelo lúdico: o brincar, a criança com deficiência física e a terapia ocupacional. 3 ed. São Paulo: Roca.

Ferreira, D. (2010). O direito a brincar. Cadernos de educação de Infância, 90,12-13, Lisboa: APEI.

Figueiredo, M., Gatinho, A., Torres, N., Pinto, A., Santos, A J. \& Veríssimo, M. (2015). Representações de vinculação e qualidade do brincar interativo em crianças em idade préescolar. Análise Psicológica, 33 (3), 335-345. Retirado de http://publicacoes.ispa.pt/index.php/ap/article/view/1007

Fombonne, E. (2018). The rising prevalence of autism. Journal of Child Psychology and Psychiatry, 59, 717-720.

Fonseca, M. E. D. \& Da Silva, A. C. D. (2015). Concepções e uso do brincar na prática clínica de terapeutas ocupacionais. Cad Ter Ocup. São Carlos, 23(3), 89-97.

Fuentes, J., Hervás, A. \& Howlin, P. (2020). ESCAP practice guidance for autism: a summary of evidence-based recommendations for diagnosis and treatment. European Child Adolescent Psychiatry.

Griesi-Oliveira, K. \& Sertie, A. L. (2017). Transtornos do espectro autista: um guia atualizado para aconselhamento genético. Einstein, São Paulo, 15 (2), 233-238.

Huizinga, J. (2019). Homo ludens: O jogo como elemento da cultura. 2 ed. São Paulo: Perspectiva.

Leal, B. S. F. M., Gradim, L. C. C. \& Souza, V. R. B. (2020). Habilidades sociais em crianças com transtorno do espectro autista: uma análise da prática em Terapia Ocupacional. Rev. Interinst. Bras. Ter. Ocup. Rio de Janeiro. 5 (6), 121-139.

Lee, G. T., Xu, S., Guo, S., Gilic, L., Pu, Y. \& Xu, J. (2019). Teaching imaginary objects symbolic play to young children with autism. J. Autism Dev Disord, 49, 4109-4122.

Loke, Y. J., Hannan, A. J. \& Craig, J. M. (2015). The role of epigenetic change in autism spectrum disorders. Front Neurol, 6 (107). DOI: 10.3389/fneur.2015.00107.

Maia, F. A., Almeida, M. T. C., Alves, M. R., Bandeira, L. V. S., Da Silva, V. B., Nunes, N. F., Cardoso, L. C. G. \& Silveira, M. F. (2018). Transtorno do espectro do autismo e idade dos genitores: estudo de caso-controle no Brasil. Caderno de Saúde Pública, 34 (8), e00109917. Epub August 20, 2018. https://dx.doi.org/10.1590/0102-311x00109917.

Mendes K.D.S., Silveira, R.C.C.P. \& Galvão, C.M. (2008). Revisão Integrativa: método de pesquisa para incorporação de evidências na saúde e na Enfermagem. Texto Contexto Enferm, 17(Supl. 4), 758-764.

Minayo, M. C. S. (2014). O desafio do conhecimento: pesquisa qualitativa em saúde. 14. ed. São Paulo: Hucitec.

Minayo, M. C. S., Deslandes, S. F. \& Gomes, R. (2013). Pesquisa social: teoria, método e criatividade. 33. ed. Petrópolis: Vozes.

Moraes, I. M. (2012). A pedagogia do brincar: Intercessões da ludicidade e da psicomotricidade para o desenvolvimento infantil. 2012. [Dissertação de Mestrado em Educação- Centro Universitário Salesiano de São Paulo - UNISAL, Americana]. https://unisal.br/wpcontent/uploads/2013/03/Disserta\%C3\%A7\%C3\%A3o-Ingrid-M-Moares.pdf.

Orr, E. (2020). Object play as a mediator of the role of exploration in communication skills development. Infant Behavior and Development, 60. DOI: 10.1016/j.infbeh.2020.101467.

Piaget, J. A. (1978). Formação do símbolo na criança: imitação jogo e sonho, imagem e representação. Rio de Janeiro: Zahar.

Potter, C. (2017). Father involvement in the care, play, and education of children with autism. Journal of Intellectual \& Developmental Disability, 42 (4), 1-10. 
Ramos, D. K., Lorenset, C. C. \& Petri, G. (2016). Jogos educacionais: contribuições da neurociência à aprendizagem. Revista $X, 2(1), 1-17$.

Santos, L. G. (2016). A importância do brincar para o desenvolvimento cognitivo da criança na educação infantil pré-escolar sob a percepção de professores. Projeção e Docência, 7(2), 23-34.

Saviani, I. \& Pinheiro, R. (2014). Formação em espaços lúdicos. São Paulo: Fundação Maria Cecilia Souto Vidigal, Coleção primeiríssima infância, 5.

Souza, J. S. (2019). Brincar em tempos de tecnologias digitais móveis. [Tese de Doutorado em Educação, Universidade Federal da Bahia, Bahia, Brasil]. https://repositorio.ufba.br/ri/bitstream/ri/28762/3/Joseilda.pdf

Souza, M.T., Silva, M.D. \& Carvalho, R. (2010). Revisão integrativa: o que é e como fazer. Einstein; 8(Supl.1), 102-106.

Vieira, M. S. (2018). O brincar de crianças em idade pré-escolar com perturbação do espectro do autismo. [Dissertação de Mestrado, Escola Superior de Educação de Lisboa, Portugal]. https://repositorio.ipl.pt/bitstream/10400.21/9529/1/Tese\%20MIP\%20$\% 20$ Melissa\%20Vieira.pdf

Vygotsky, L. S. (2008). A brincadeira e o seu papel no desenvolvimento psíquico da criança. Revista Virtual de Gestão de Iniciativas Sociais, 23-36.

Who. World Health Organization. Factsheet: Autism spectrum disorders 2019. Recuperado de https://www.who.int/news-room/fact-sheets/detail/autism-spectrum-disorders. 